\title{
Hypomethylation effects of curcumin, demethoxycurcumin and bisdemethoxycurcumin on WIF-1 promoter in non-small cell lung cancer cell lines
}

\author{
YU-LIANG LIU, HE-PING YANG, LIANG GONG, CHUN-LAN TANG and HAI-JING WANG
}

Department of Respiratory Medicine, Southwest Hospital, The Third Military Medical University, Chongqing, P.R. China

Received November 29, 2010; Accepted April 6, 2011

DOI: $10.3892 / \mathrm{mmr} .2011 .473$

\begin{abstract}
The tumor suppressor gene Wnt inhibitory factor-1 (WIF-1) has been found to be promoter hypermethylated and silenced in lung cancer cell lines and tissues. Curcuminoids are major active components of the spice turmeric, and have recently been reported to be potential hypomethylation agents. In the present study, the hypomethylation effects of three major curcuminoids, curcumin, demethoxycurcumin and bisdemethoxycurcumin, were compared in vitro using ELISA, and their demethylation potential was confirmed by methylation-specific PCR. It was found that bisdemethoxycurcumin possesses the strongest demethylation function in vitro compared to the other two curcuminoids, exerting its effect at a minimal demethylation concentration of $0.5-1 \mu \mathrm{M}$. The WIF-1 promoter region was demethylated after treatment with $20 \mu \mathrm{M}$ demethoxycurcumin and bisdemethoxycurcumin, but failed to respond to $20 \mu \mathrm{M}$ curcumin. In the A549 cell line, RT-PCR and Western blotting were used to confirm that WIF-1 expression was restored after curcuminoid-induced promoter hypermethylation. Since the results regarding the demethylation potential of the three major curcuminoids to restore WIF-1 expression indicated that bisdemethoxycurcumin has the strongest hypomethylation effect, this curcuminoid may have therapeutic use in the restoration of WIF-1 expression in NSCLC.
\end{abstract}

\section{Introduction}

Lung cancer is the leading cause of cancer-related death worldwide. It is estimated that in 2010, more than 157,300 individuals succumbed to the disease in the US (1). Several known and putative tumor suppressor genes have been found

Correspondence to: Dr He-Ping Yang, Department of Respiratory Medicine, Southwest Hospital, The Third Military Medical University, Gaotanyan, Sha-Ping-Ba, Chongqing 400038, P.R. China E-mail: hepingyang@gmail.com

Key words: curcuminoids, lung neoplasms, promoter demethylation, Wnt inhibitory factor-1 to be frequently inactivated by promoter methylation in lung cancer (2-4). Wnt inhibitory factor-1 (WIF-1) is one of those silenced genes (5).

WIF-1 is located at chromosome $12 \mathrm{q} 14$ and contains an $\mathrm{N}$-terminal signal sequence, an N-terminal domain of -150 amino acids, five epidermal growth factor-like repeats, and a C-terminal hydrophilic domain (6). WIF-1 is a highly conserved gene involved in the regulation of the Wnt pathway as an inhibitory factor binding Wnt ligands. Aberrant Wnt activation has been implicated in various types of human cancer, including colorectal cancer, melanoma and leukemia, as well as nonsmall cell lung cancer (NSCLC) (7-10). Unlike in colorectal cancer, in NSCLC unrestricted Wnt ligands are responsible for Wnt rampage, rather than genetic mutations of APC or $\beta$-catenin (10). Accompanying the unrestricted Wnt ligands is the downregulation of WIF-1, which has been observed in several types of human cancer, including prostate, breast, lung, bladder, melanoma and gastrointestinal malignancies (11-13). Recently, WIF-1 silencing due to its promoter hypermethylation was demonstrated in NSCLC, astrocytomas and nasopharyngeal cancer $(4,14,15)$. Thus, the suppressed expression of WIF-1 due to its promoter hypermethylation may contribute to the upregulation of Wnt signaling in NSCLC. Re-expression of WIF-1 may therefore result in the downregulation of the Wnt pathway and the inhibition of cancer cell growth.

Hypomethylation agents are natural inhibitors of DNA methyltransferases (DnMT) that have the potential to reverse the promoter hypermethylation of WIF-1. Several categories of DnMT1 inhibitors have been identified in previous studies. Among these, nucleoside analogs such as decitabine or 5-azacytidine appear to be most effective in inducing global DNA hypomethylation and the reactivation of epigenetically silenced tumor suppressor genes in malignant cells (16-18). However, toxicity (e.g., myelosuppression) inherent to the cell cycle phase specificity of nucleoside analogs significantly limits the use of these drugs, particularly in patients with solid tumors (19). Thus, novel effective hypomethylating agents with a more favorable toxicity profile are urgently required.

Curcumin mixture, a yellow pigmented substance isolated from the rhizome of turmeric, is proven to have a variety of medicinal properties, including anti-inflammatory, antibacterial, antifungal, antioxidant, anticarcinogen, antimutagen, and anticancer effects (20-23). This active extract can be subdivided 
into three distinct components: curcumin (component 1), demethoxycurcumin (DMC; component 2) and bisdemethoxycurcumin (BDMC; component 3) (24). Recently, computer modeling verified by DNA global hypomethylation in leukemia cell lines was used to demonstrate that curcumin mixture or monomers are potential hypomethylating agents (25). In the present study, we further investigated the hypomethylation potential of these three curcuminoids, and examined and compared their potential to restore WIF-1 expression in NSCLC cell lines.

\section{Material and methods}

Chemicals. RPMI-1640, fetal bovine serum (FBS), penicillin and streptomycin were purchased from Gibco-Invitrogen (MI, USA). Small airway epithelial cell basal medium (SAGM) was purchased from Lonza (Walkersville, MD, USA). Curcumin, DMC and BDMC were purchased from TCI (Tokyo, Japan). 5-Aza-2'-deoxycytidine (DAC) was obtained from SigmaAldrich (St. Louis, MO, USA). PNPP substrate was purchased from Thermo Fisher Scientific (Rockford, IL, USA). DnMT1 and S-adenosyl methionine (SAM) were purchased from New England Biolabs (Beverly, MA, USA), HapII was obtained from Takara (Dalian, China). RNA and DNA extraction reagents and purification kits were acquired from Tiangen Biotech (Beijing, China). Bisulfite modification of genomic DNA was performed using the EZ DNA methylation kit (Zymo Research, Orange, CA, USA). Takara Ex Taq polymerase was used for DNA amplification (Takara, China). Anti-human WIF-1 antibody was purchased from Abcam (Cambridge, UK), Goat anti-mouse IgG horseradish peroxidase-conjugated secondary antibody was from Zhongshan Goldenbridge Biotechnology (Beijing, China). Anti-digoxigenin-AP antibody was purchased from Roche (Mannheim, Germany). Other reagents used were of analytical grade and procured locally.

Cell culture and chemical treatment. The NSCLC cell lines A549, H460 and SPC-A-1 were used in this study, and routinely maintained at our laboratory. The cells were cultured in RPMI 1640 supplemented with $10 \%$ fetal bovine serum, penicillin $(100 \mathrm{U} / \mathrm{ml})$ and streptomycin $(100 \mu \mathrm{g} / \mathrm{ml})$. The lung epithelial cell line SAEC was obtained from Clonetics (San Diego, CA, USA) and maintained in SAGM supplemented with $10 \%$ fetal bovine serum, penicillin $(100 \mathrm{U} / \mathrm{ml})$ and streptomycin $(100 \mu \mathrm{g} / \mathrm{ml})$. The cells were grown at $37^{\circ} \mathrm{C}$ in a humidified atmosphere of $5 \% \mathrm{CO}_{2}$. To evaluate the hypomethylation effect of bisdemethoxycurcumin, DAC was used as positive control. Curcumin, DMC, BDMC and DAC were prepared as stock solutions $(20 \mathrm{mM})$ in dimethyl sulfoxide (DMSO) and diluted in sterile-filtered media before use. Cells $\left(1 \times 10^{5}\right)$ were seeded into 6-well culture plates. After $24 \mathrm{~h}$ of culture, the cells were treated with curcumin, DMC, BDMC $(0-100 \mu \mathrm{M})$ or DAC $(20 \mu \mathrm{M})$ for $72 \mathrm{~h}$.

ELISA. As a detection probe, a 38-bp double strand (ds)oligonucleotide labeled with 3 '-biotin in one strand and 3'-digoxigenin in its complementary strand was designed according to the WIF-1 promoter region from -407 to -445 bp (5'-GGTCCCTCGCGGCCCCGCCCCCGGGGGGGCCCC ACAGC-3') and synthesized by Sangon Biotech. This oligo- nucleotide was loaded onto streptavidin plates followed by buffer containing DnMT1 $(2,000 \mathrm{U} / \mathrm{ml})$ and SAM $(160 \mu \mathrm{M})$, and then incubated for $10 \mathrm{~min}$ at $37^{\circ} \mathrm{C}$. Various concentrations $(1,10,50,100$ and $500 \mathrm{nM}$, or 1, 10, 30, 50, 70 and $100 \mu \mathrm{M})$ of the three curcuminoids were then added, along with the endonuclease HapII (100 U/ml). The components were incubated for $1 \mathrm{~h}$ at $37^{\circ} \mathrm{C}$. Anti-digoxigenin-AP antibody was added to the plates along with PNPP substrate (dissolved according to the manufacturer's instructions), followed by incubation at room temperature for $30 \mathrm{~min}$. $\mathrm{NaOH}(1 \mathrm{~mol} / \mathrm{l})$ was added to each well to stop the reaction. Non-probe plated wells treated by the same procedure served as blank controls, and wells loaded with DAC were usde as positive controls. The absorbance was measured at $405 \mathrm{~nm}$ in a kinetic ELISA reader (Synergy $^{\mathrm{TM}}$ 2, Biotek, VT, USA). The experiments with each concentration of the tested reagents were repeated in triplicate wells.

$R T-P C R$. Total RNA from the lung cancer cell lines was isolated using the RNAprep pure cell/bacteria kit. Reverse transcription-PCR (RT-PCR) was performed in a Bio-Rad MyCycler $^{\text {TM }}$ thermal cycler using a two-step RT-PCR kit from Promega Biotech Co., Ltd. according to the manufacturer's instructions. Primers for RT-PCR were obtained from Sangon Biotech Co., Ltd. (Shanghai, China). Primer sequences for WIF-1 were 5'-CCGAAATGGAGGCTTTTGTA-3' (forward) and 5'-TGGTTGAGCAGTTTGCTTTG-3' (reverse) (4). GAPDH (glyceraldehyde-3-phosphate dehydrogenase) was used as an internal control.

Western blotting. Total protein from the NSCLC cancer cell lines was extracted using standard protocols. The protein concentrations were determined using the BCA protein assay (Hyclone-Pierce, South Logan, UT, USA). Proteins $(50 \mu \mathrm{g})$ were electrophoresed on a $12 \%$ SDS polyacrylamide gel and transferred onto polyvinylidene fluoride (PVDF) membranes. The membranes were blocked in $5 \%$ nonfat dry milk in Tris-buffered saline (TBS) for $1 \mathrm{~h}$ at room temperature, then incubated with anti-WIF-1 (diluted 1:50-1:200) antibody overnight at $4{ }^{\circ} \mathrm{C}$. After washing three times with Tris-buffered saline containing Tween-20 (TBST), the membranes were incubated with anti-mouse IgG horseradish peroxidase-conjugated antibody (diluted 1:2,000) for $2 \mathrm{~h}$ at room temperature. Each sample was also probed with an anti-GAPDH antibody (Santa Cruz Biotechnology) as a loading control. The membranes were then washed three times with TBST, and the protein signals were detected using the ECL Western blotting system (Beyotime). The relative amount of protein on the blots was determined by densitometry using Quantity One quantization software (Bio-Rad, Munich, Germany).

Methylation-specific PCR. Bisulfite-treated genomic DNA was amplified using either a methylation-specific or a nonmethylation-specific primer set (4). 2X PCR Master Mix (Tiangen) was used in the experiments. Methylation-specific primers sequences were 5'-GGGCGTTTTATTGGGCGTAT-3' (forward) and 5'-AAACCAACAATCAACGAAC-3' (reverse). Non-methylation-specific primer sequences were 5'-GGGTGT TTTATTGGGTGTAT-3' (forward) and 5'-AAACCAACAATC AACAAAAC-3' (reverse). 


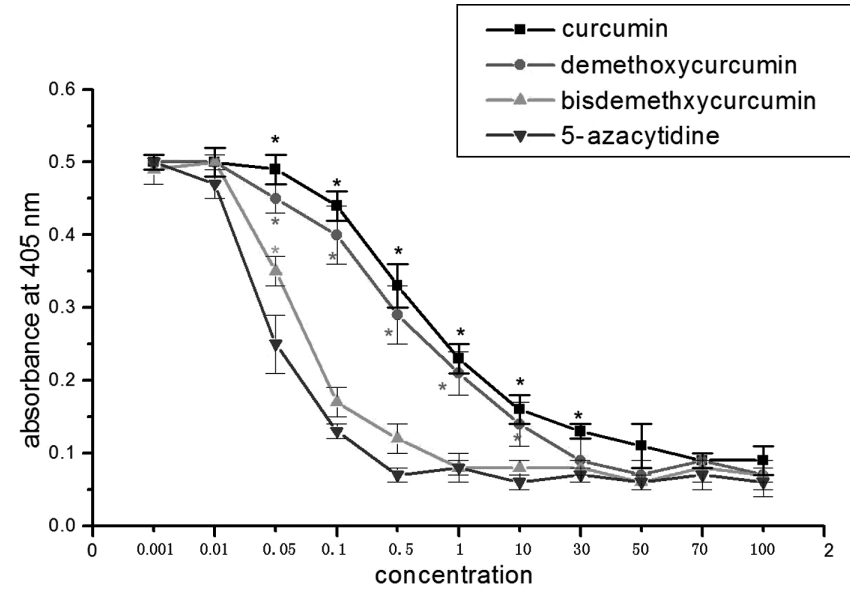

Figure 1. The inhibitory effect of curcumin $(\boldsymbol{\bullet}), \operatorname{DMC}(\bullet), \operatorname{BDMC}(\triangle)$ and DAC $(\boldsymbol{\nabla})$ on DnMT1 activity. The absorbance at $405 \mathrm{nM}$ was detected for various concentrations of curcumin, DMC, BDMC or DAC, and the concentration required to reach a plateau was defined as the minimal demethylation concentration (MDC). ${ }^{*} \mathrm{p}<0.01$ compared to the DAC-treated (positive control) cells.

\begin{tabular}{|c|c|c|c|c|c|c|c|}
\hline SAEC & & $\mathrm{H}^{4}$ & & A5 & & & \\
\hline $\mathrm{U}$ & $M$ & $\mathrm{U}$ & $M$ & $\mathrm{U}$ & $M$ & $\mathrm{U}$ & $M$ \\
\hline$=$ & & & 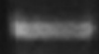 & & 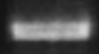 & & 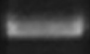 \\
\hline
\end{tabular}

Figure 2. Methylation-specific PCR analysis of the WIF-1 promoter region in the H460, A549 and SPC-A-1 NSCLC cell lines. Lane U, unmethylated DNA product with non-methylation-specific primers; lane M, methylated DNA product amplified with methylation-specific primers. The normal lung epithelial cell line SAEC served as a control.

Statistical analysis. Data are expressed as the mean \pm standard deviation (SD) from three independent experiments conducted in triplicate. Statistical differences between the means were tested using one-way ANOVA. $\mathrm{p}<0.05$ was considered to be statistically significant.

\section{Results}

Differential hypomethylation potential of curcumin, DMC and BDMC. The concentration of the oligonucleotide probe added was adjusted to a suitable concentration by standard curve according to the Beer-Lambert law; a concentration of $1 \mu \mathrm{g} /$ well was selected for this experiment. The oligonucleotide probe contained a CCGG sequence, which, when cleaved by HapII, resulted in a loss of the 3'-digoxigenin. When anti-digoxigenin-AP antibody and the substrate PNPP were added to the solution, no signal was detected at $405 \mathrm{nM}$. By contrast, when the CCGG sequence of the ds-oligonucleotide was methylated to $\mathrm{CCmGG}$, it resulted in resistance to the cleavage activity of HapII, maintenance of the 3'-digoxigenin of the ds-oligonucleotide and, in turn, signal acquisition under the same condition. The methylation level of the ds-oligonucleotide was directly correlated with the intensity of the assay signal, and in turn with the enzymatic activity of DnMT1. Thus, the hypomethylation effects of the three curcuminoids

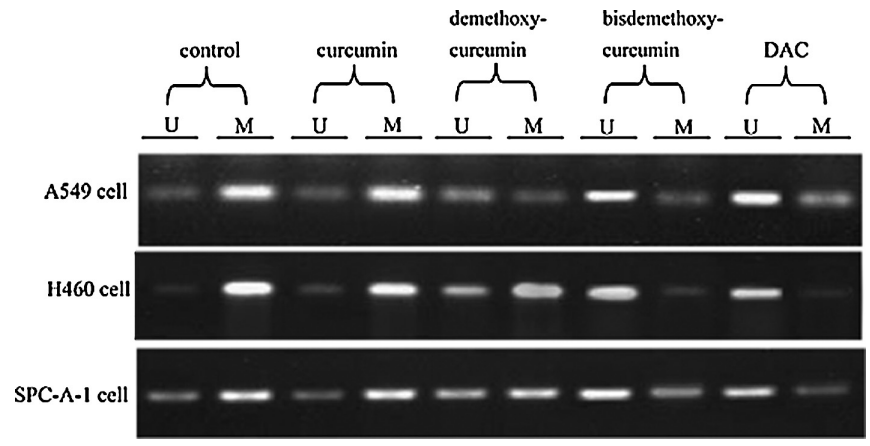

Figure 3. Methylation-specific PCR analysis of the WIF-1 promoter region in the A549, H460 and SPC-A-1 cell lines after treatment with various concentrations of curcumin, DMC, BDMC or DAC for $72 \mathrm{~h}$. DAC was used as a positive demethylation control. Lanes U, unmethylated DNA product amplified with non-methylation-specific primers; lanes M, methylated DNA product amplified with methylation-specific primers.

were evaluated and compared in terms of their absorbance intensity. DAC, a traditional hypomethylation agent, served as the positive control.

It was found that as the concentration of the three curcuminoids increased, the absorbance at $405 \mathrm{nM}$ decreased, indicating that the hypomethylation effect of the curcuminoids was dose-dependent. The component which decreased the absorbance intensity to baseline levels ( $\sim 1$ that determined by the standard curve without the soligonucleotide probe) at the lowest applied concentration was determined to be the most effective hypomethylation agent. The hypomethylation potential of curcumin, DMC and BDMC was then compared to this standard. BDMC completely dissipated the methylation of the CCGG sequence of the probe at a concentration of $0.5-1 \mu \mathrm{M}$, while curcumin and DMC had the same effect only at $10 \mu \mathrm{M}$ and $5 \mu \mathrm{M}$, respectively (Fig. 1).

WIF-1 methylation in A549, H460 and SPC-A-1 cells. To address the ability of the curcuminoids to reverse the hypermethylation of WIF-1, WIF-1 methylation in the NSCLC cell lines A549, H460, and SPC-A-1 was initially investigated. As previously reported (4), the promoter hypermethylation of WIF-1 was verified in the A549, H460 and SPC-A-1 cell lines using methylation-specific PCR, while normal human small airway epithelial cells were determined to be hypomethylated (Fig. 2).

WIF-1 promoter demethylation by curcuminoids in A549, H460 and SPC-A-1 cells. Although BDMC was shown to have a potential hypomethylation effect in vitro at a concentration of $1 \mu \mathrm{M}$, in the cultured cells it had to be to transferred into the cytoplasm, for which a higher concentration was required. As a result, all the NSCLC cell lines were treated with a non-cytotoxic concentration of $20 \mu \mathrm{M}$ curcumin, DMC or BDMC for 3 days, with DAC as a positive control. The WIF-1 promoter region of the A549, H460 and SPC-A-1 cell lines is generally hypermethylated. Curcumin failed to show any significant hypomethylation effect at the tested concentration. However, DMC and BDMC exhibited a hypomethylation effect, in that they increased the unmethylated PCR product while decreasing the methylated PCR product. The hypometh- 
A

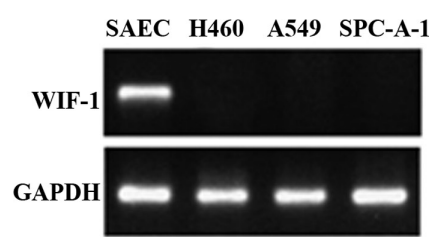

B

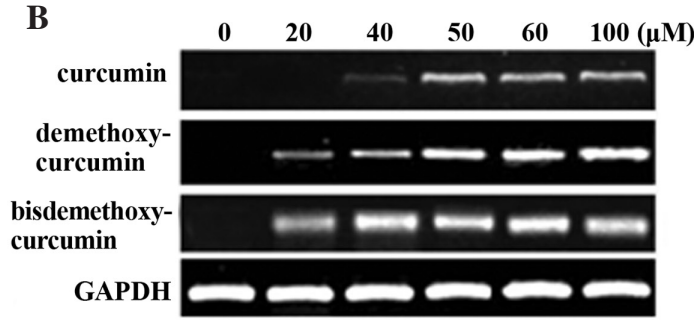

C

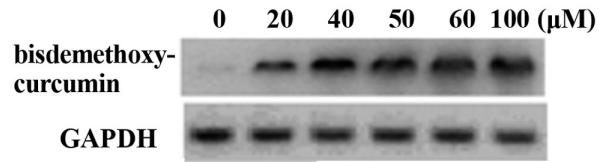

Figure 4. Upregulation of WIF-1 after curcumin, DMC or BDMC treatment. (A) Reverse transcription-PCR results from the A549, H460 and SPC-A-1 NSCLC cell lines. The 188-bp fragment of human WIF-1 cDNA is amplified. A GAPDH-specific probe was used as an internal control. SAEC cells served as the control. (B) A549 cells were treated with various concentrations of curcumin, DMC or BDMC for $72 \mathrm{~h}$. RT-PCR was performed to investigate the mRNA level of WIF-1. (C) Western blotting was performed to investigate the protein level of WIF-1. GAPDH was used as an internal control.

ylation effect of BDMC was particularly strong, comparable to that of DAC (Fig. 3).

Restoration of WIF-1 mRNA and protein expression in A549 cells. Curcumin showed a hypomethylation effect in vitro but failed to induce WIF-1 promoter demethylation at a concentration of $20 \mu \mathrm{M}$ in cultured cells, suggesting that the concentration used was insufficient. To evaluate the effect of curcumin, DMC and BDMC on the restoration of WIF-1 expression in the A549 cell line, an enhanced concentration of the three curcuminoids, ranging from 20 to $100 \mu \mathrm{M}$, was used in the following experiment.

WIF-1 mRNA was detected in the tested cell lines, and the WIF-1 gene was found to be downregulated in the NSCLC cells (Fig. 4A). This supports the conclusions of previous studies, that hypermethylation of the WIF-1 promoter may lead to the suppression of WIF-1 expression. The levels of WIF-1 mRNA (after normalization to GAPDH) in the A549 cells were restored after treatment with the curcuminoids. However, curcumin only began to reactivate WIF-1 expression at a concentration of $40 \mu \mathrm{M}$, while DMC and BDMC reactivated WIF-1 expression at a concentration of $20 \mu \mathrm{M}$ (Fig. 4B). The levels of WIF-1 protein detected in the A549 cells in response to BDMC treatment were in agreement with the results of RT-PCR, suggesting a strong effect on WIF-1 restoration by BDMC in the A549 cell line (Fig. 4C).

\section{Discussion}

The aberrant methylation of $\mathrm{CpG}$ islands in the promoter region of genes has been associated with the transcriptional inactivation of tumor suppressor genes in human cancer. This epigenetic process is considered to be the major gene silencing mechanism for tumor suppressor genes, along with genetic alterations such as mutations and deletions (26,27). A growing number of genes have been identified to show abnormal $\mathrm{CpG}$ island promoter methylation in lung cancer, many of which are tumor suppressor genes.

WIF-1 is a prominent case of tumor suppressor gene promoter methylation in NSCLC. WIF-1 inhibits Wnt signaling by directly binding to Wnt molecules, downregulating the Wnt pathway and inhibiting NSCLC cell growth. However, WIF-1 is silenced by promoter methylation in primary tumors and in many NSCLC cell lines. Although transfection with WIF-1 induces apoptosis in NSCLC cells and also inhibits NSCLC tumor xenograft growth (28), restoring WIF-1 expression using hypomethylation agents is more convenient and economic than gene transfection.

Hypomethylation agents can be roughly classified into two categories according to the way in which they interact with DNA methyltransferases (DnMTs) (24). Nucleoside analogs such as 5-azacytidine and zebularine covalently trap DnMT1 through incorporation into DNA, while non-nucleoside analogs inhibit DnMT through non-covalent blocking of the DnMT catalytic site or by other mechanisms. The dilemma of epigenetic targeting therapy is the high toxicity of effective nucleoside analogs and the low potency of non-nucleoside analogs. Finding a non-nucleoside hypomethylating agent that can covalently block DnMT without incorporation into DNA may reduce toxicity and guarantee potency.

Curcumin compound, a naturally occurring yellow pigment isolated from turmeric, is a well-known antitumoral agent. Recent studies based on computer modeling also suggest that curcumin is a hypomethylation agent. It has been suggested that $\alpha, \beta$-unsaturated carbonyl moieties of curcuminoids may covalently block DnMT1 depending on their conformation (24). However, to date there has been no indication of which component of turmeric has the strongest suppressive effect on DnMT1 activity, nor has their potential to reactivate tumor suppressor genes been investigated.

In the present study, the hypomethylation effect of curcumin, DMC and was were quantified according to their ability to suppress DnMT1 activity. It was found that 0.5-1 $\mu \mathrm{M}$ of BDMC completely inhibited DnMT1 activity, while the minimal requirement for curcumin and DMC was $10 \mu \mathrm{M}$ and $5 \mu \mathrm{M}$, respectively. The minimal concentration of the postive control DAC required to suppress DnMT1 was around $0.1-0.5 \mu \mathrm{M}$. These results suggest that the hypomethylation potential of these three curcuminoids should be ordered as $\mathrm{BDMC}>\mathrm{DMC}>$ curcumin. In addition, BDMC has a strong demethylation effect, comparable to that of DAC. We further investigated and compared the hypomethylation effect of curcumin, DMC and BDMC on the WIF-1 promoter, and determined their ability to restore WIF-1 expression.

In the present study, WIF-1 promoter region methylation and gene expression was detected in the A549, H460 and SPC-A-1 NSCLC cell lines. As expected, the promoter region of WIF-1 in the cell lines was highly methylated and the gene was completely silenced. This supports the findings of previous studies that the WIF-1 gene is generally suppressed 
in NSCLC tumor tissues and cell lines. After being treated with $20 \mu \mathrm{M}$ of curcuminoids, the morphology and viability of the NSCLC cells did not change as compared to those of the vehicle control. This suggests that curcuminoids at a concentration of $20 \mu \mathrm{M}$ do not alter the morphology and viability of NSCLC cell lines. When treated with $20 \mu \mathrm{M}$ of curcumin, no significant promoter hypomethylation was observed in NSCLC cell lines. In addition, WIF-1 mRNA was not detected in these cell lines. To restore WIF-1 mRNA with curcumin, it was necessary to enhance the concentration to $40 \mu \mathrm{M}$, and even at this concentration WIF-1 expression was only partially restored. When treated with $20 \mu \mathrm{M}$ of DMC and BDMC, the promoter hypomethylation effect of WIF-1 was clearly observable, and WIF-1 mRNA levels were restored. Consistent with the results of the ELISA assay, BDMC showed the strongest hypomethylation effect, as more unmethylated PCR products and more WIF-1 mRNA were detected. The results clearly indicate that BDMC, the minor component of curcuminoid powder, is the most effective component in restoring WIF-1 gene expression, followed in decreasing order by DMC and curcumin. This finding, that BDMC suppressed the cell proliferation of NSCLC cells most effectively, is similar to results from a previous study conducted by our group (29).

This study is the first to provide evidence of a differential hypomethylation effect of the three curcuminoids and their potential to reactivate WIF-1 from a silenced state by methylation. This suggests a potential therapeutic use for curcuminoids in the reversal of WIF-1 promoter methylation and restoration of WIF-1 expression in NSCLC.

\section{Acknowledgements}

The authors wish to thank Dr Bin Wang, Pathology Department of Southwest Hospital, Dr Zhen-Ning Tang, Department of Breast Surgery, Southwest Hospital and Dr Yu Xu, Department of Oncology, Xinqiao Hospital, for their keen interest and assistance with this study.

\section{References}

1 American Cancer Society: Cancer Facts and Figures 2010. American Cancer Society, Atlanta, 2010.

2. Tessema M, Willink R, Do K, et al: Promoter methylation of genes in and around the candidate lung cancer susceptibility locus 6q23-25. Cancer Res 68: 1707-1714, 2008.

3. Glazer CA, Smith IM, Ochs MF, et al: Integrative discovery of epigenetically derepressed cancer testis antigens in NSCLC. PLoS One 4: e8189, 2009.

4. Sun W, Iijima T, Kano J, et al: Frequent aberrant methylation of the promoter region of sterile alpha motif domain 14 in pulmonary adenocarcinoma. Cancer Sci 99: 2177-2184, 2008.

5. Mazieres J, He B, You L, et al: Wnt inhibitory factor-1 is silenced by promoter hypermethylation in human lung cancer. Cancer Res 64: 4717-4720, 2004.

6. Batra S, Shi Y, Kuchenbecker KM, et al: Wnt inhibitory factor-1, a Wnt antagonist, is silenced by promoter hypermethylation in malignant pleural mesothelioma. Biochem Biophys Res Commun 342: 1228-1232, 2006.

7. Vermeulen L, De Sousa EMF, van der Heijden M, et al: Wnt activity defines colon cancer stem cells and is regulated by the microenvironment. Nat Cell Biol 12: 468-476, 2010.
8. Chien AJ, Moore EC, Lonsdorf AS, et al: Activated Wnt/ beta-catenin signaling in melanoma is associated with decreased proliferation in patient tumors and a murine melanoma model. Proc Natl Acad Sci USA 106: 1193-1198, 2009.

9. Tickenbrock L, Hehn S, Sargin B, et al: Activation of Wnt signalling in acute myeloid leukemia by induction of Frizzled-4. Int J Oncol 33: 1215-1221, 2008.

10. Mazieres J, He B, You L, Xu Z and Jablons DM: Wnt signaling in lung cancer. Cancer Lett 222: 1-10, 2005.

11. Wissmann C, Wild PJ, Kaiser S, et al: WIF1, a component of the Wnt pathway, is down-regulated in prostate, breast, lung, and bladder cancer. J Pathol 201: 204-212, 2003.

12. Byun T, Karimi M, Marsh JL, Milovanovic T, Lin F and Holcombe RF: Expression of secreted Wnt antagonists in gastrointestinal tissues: potential role in stem cell homeostasis. J Clin Pathol 58: 515-519, 2005

13. Veeck J, Wild PJ, Fuchs T, et al: Prognostic relevance of Wnt-inhibitory factor-1 (WIF1) and Dickkopf-3 (DKK3) promoter methylation in human breast cancer. BMC Cancer 9: 217, 2009.

14. Yang Z, Wang Y, Fang J, Chen F, Liu J and Wu J: Expression and aberrant promoter methylation of Wnt inhibitory factor-1 in human astrocytomas. J Exp Clin Cancer Res 29: 26, 2010.

15. Lin YC, You L, Xu Z, et al: Wnt signaling activation and WIF-1 silencing in nasopharyngeal cancer cell lines. Biochem Biophys Res Commun 341: 635-640, 2006.

16. Christman JK: 5-Azacytidine and 5-aza-2'-deoxycytidine as inhibitors of DNA methylation: mechanistic studies and their implications for cancer therapy. Oncogene 21: 5483-5495, 2002.

17. Schneider-Stock R, Diab-Assef M, Rohrbeck A, et al: 5-Aza-cytidine is a potent inhibitor of DNA methyltransferase $3 \mathrm{a}$ and induces apoptosis in HCT-116 colon cancer cells via Gadd45- and p53-dependent mechanisms. J Pharmacol Exp Ther 312: 525-536, 2005

18. Brueckner B, Rius M, Markelova MR, et al: Delivery of 5-azacytidine to human cancer cells by elaidic acid esterification increases therapeutic drug efficacy. Mol Cancer Ther 9: 1256-1264, 2010.

19. Graham JS, Kaye SB and Brown R: The promises and pitfalls of epigenetic therapies in solid tumours. Eur $\mathrm{J}$ Cancer 45: 1129-1136, 2009

20. Chainani-Wu N: Safety and anti-inflammatory activity of curcumin: a component of tumeric (Curcuma longa). J Altern Complement Med 9: 161-168, 2003.

21. Mishra S, Narain U, Mishra R and Misra K: Design, development and synthesis of mixed bioconjugates of piperic acid-glycine, curcumin-glycine/alanine and curcumin-glycine-piperic acid and their antibacterial and antifungal properties. Bioorg Med Chem 13: 1477-1486, 2005.

22. Sa G and Das T: Anti cancer effects of curcumin: cycle of life and death. Cell Div 3: 14, 2008.

23. Aggarwal BB, Kumar A and Bharti AC: Anticancer potential of curcumin: preclinical and clinical studies. Anticancer Res 23: 363-398, 2003

24. Yodkeeree S, Chaiwangyen W, Garbisa S and Limtrakul P: Curcumin, demethoxycurcumin and bisdemethoxycurcumin differentially inhibit cancer cell invasion through the downregulation of MMPs and uPA. J Nutr Biochem 20: 87-95, 2009.

25. Liu Z, Xie Z, Jones W, et al: Curcumin is a potent DNA hypomethylation agent. Bioorg Med Chem Lett 19: 706-709, 2009.

26. Saito T, Oda Y, Kawaguchi K, et al: Possible association between tumor-suppressor gene mutations and hMSH2/hMLH1 inactivation in alveolar soft part sarcoma. Hum Pathol 34: 841-849, 2003.

27. Kang JU, Koo SH, Kwon KC and Park JW: Frequent silence of chromosome 9p, homozygous DOCK8, DMRT1 and DMRT3 deletion at 9p24.3 in squamous cell carcinoma of the lung. Int J Oncol 37: 327-335, 2010.

28. Kim J, You L, Xu Z, et al: Wnt inhibitory factor inhibits lung cancer cell growth. J Thorac Cardiovasc Surg 133: 733-737, 2007.

29. Yang HP, Gong L, Tang CL, Li JM, and Wang HJ: A study of bisdemethoxycurcumin and its effects on the biological properties of human pulmonary adenocarcinoma cell line A549. Chongqing Med J (in Chinese) 34: 868-869, 2005. 